\title{
5
}

\section{Non Resonant Microwave Absorption (NRMA) Anomalies in High Temperature Superconductors (HTS) Relevance of Electromagnetic Interactions (EMI) and Energy Stabilized Josephson (ESJ) Fluxons}

\author{
G.K. Padam \\ Materials Physics and Engineering Division, National Physical Laboratory, New Delhi, \\ India
}

\section{Introduction}

High temperature superconductors (HTS) due to their higher operating temperatures, 2dimensional (2D) layered structure and very short coherence length $(\xi)$ are known to show some interesting properties especially magnetic field dependent microwave (MW) absorption effects at very low magnetic fields of the order of zero gauss; Kadam et al.1987; Blazy et al.1987, 1988, 1989;) while carrying out energy /absorption studies at MW frequencies. One striking example of this is the appearance of very strong non-resonant microwave absorption (NRMA) signal centered around zero magnetic field in the superconducting state at near below $\mathrm{T}_{\mathrm{c}}$ in cuprate based HTS :YBCO, reported by Bhat et al.(1987) using conventional electron spin resonance (ESR) spectrometer. This was followed by reports on other cuprate based HTS (Portis et al.,1987, Dulcic et al.1987,1988,1989,1990; Pakulis \& G.V.Chandershekar,1989; Sugawara et al., 2000;Hashmizume et al.2001; Padam et al.,1999) and also on a different variety of superconductors like: borocarbides (Kadam et al.,1994), oxyflorides (Kadam et al.,1995) alkali fullerides (Roberts,1998-II), granular conventional low- $\mathrm{T}_{\mathrm{c}}$ typeI $[\mathrm{Pb}, \mathrm{Al}, \mathrm{Sn}, \mathrm{In}$ etc.]/type II [ $\mathrm{Nb}, \mathrm{Nb}_{3} \mathrm{Sn}, \mathrm{Nb}_{3} \mathrm{Ge} / \mathrm{PbMoS}_{6} \mathrm{O}_{8}$ etc.] (Kheifetsand et al., 1990; Blazey et al.1988,1989; Suss et al,1989;Kataev et al.,1991;Baranowsky et al.1991;Moorjani et al.1990; Bele et al.1994; Blazey et al 1987; Veinger et al. 1995), $\mathrm{MgB}_{2}$ (S.Sarangi \& S.V.Bhat, Joshi et al.,2002) including newly discovered iron pniticide (Panarina et al. 2010; Pascher et al.2010 etc. ).Before coming to the actual problem, what follows is a brief introduction of the NRMA studies done before the advent of HTS.

It is to note that the NRMA response using ESR technique was reported for the first time in 1970s by Indovina et al. in the case of granular thin films of conventional type I superconductor: $\mathrm{Pb}$. Similar results were then reported in other type I superconductors: $\mathrm{Sn}$ (Kim et al., 1972) and $\mathrm{Al}$ (Muller et al., 1980) at magnetic fields varying from few hundred gauss to many thousand gauss. These reports strongly pointed out that the NRMA signal appears only if Josephson junctions (JJs) are present and their thickness ( $t$ ) is $\geq \xi$ and it does 
not appear in JJs free superconductors and/or superconductors having very thin JJs. These JJs are the non-superconducting regions separating (i) microscopic metal superconducting grains and/or (ii) superconducting regions within the grain. The main mechanism of MW power absorption was assigned to: resistance of JJs (Kim et al.,1972; Muller et al.1980) and/or viscous motion of fluxons penetrated at JJs (Kim et al., 1972; Gittleman \& B.Rosenblum,1966) It is to be pointed out that in both these models, JJs of intergranular type (here after called as Jjinter are considered.

Despite these reports on the observation of NRMA revealing an important and direct information related with manifestation of $\mathrm{JJ}_{\text {inter }}$ in granular conventional superconductors which cannot be rendered by resistivity and specific heat measurements, not much literature on NRMA studies was available at that time. It was until the advent of HTS which are intrinsically granular in nature and showed a very strong NRMA signal at nearly zero magnetic field, NRMA became a valuable, informative and indispensable tool for examining all kinds of granular superconductors existing till today from conventional (both type I and type II) to recently discovered iron pniticide etc. particularly for the detection, variety and behavior of JJs and to know the mechanism of MW absorption.

Further, to explain the origin of NRMA signal in HTS (Portis et al., 1988) and Dulcic et al.1889) extended the previous models for conventional granular superconductors in which $J_{\text {inter }}$ play the dominant role (Indovina et al.,1970;Kim et al.1972; Muller et al.1980;Gittleman \& B.Rosenblum, 1966). This was done by including the contribution from intragranular JJs ( $\left.\mathrm{JJ}_{\text {intra }}\right)$ also. As is known that unlike in the granular conventional superconductors where, the effect of $J J_{\text {intra }}$ will be suppressed due to large $\xi$, in HTS due to very short $\xi$, the effect of $\mathrm{JJ}_{\text {intra }}$ can be easily observed. Although these models considered contribution from both the JJs $\left(\mathrm{JJ}_{\text {inter }}\right.$ and $\mathrm{JJ}_{\text {intra }}$ ), however, they associated to different mechanism of absorption. That is, Portis et al. associated NRMA to damped fluxon (intergranular fluxons at $\mathrm{JJ}_{\text {inter }}$ / intragranular fluxons at $\mathrm{JJ}_{\mathrm{sintra}}$ ) motion, whereas Dulcic et al. associated to $\mathrm{JJ}_{\text {inter }}$ and $\mathrm{JJ}_{\text {intra }}$ resistance to the induced boundary currents (Josephson currents).Further, it was pointed out by Portis et al. (1988) "although we associate the microwave absorption with damped fluxon motion, it may be equivalent to associate the absorption with Josephson currents as others have done".

The continuous growth in this field of NRMA studies, along with the extreme sensitivity and expedition of the NRMA technique apart from its ability to detect the phase transition to the superconducting state, it has opened a new window (i) to unambiguously discover/detect new superconducting phases present even in quantity as low as of the order of sub micrograms, (ii) to distinguish multiple superconducting phases in a single matrix, (iii) to tell something about the quality of the superconductor, (iv) to study a new type of device for example: a single chip comprising of a combination of insulating, semiconducting, and superconducting layers, (v) to study the phase diagram of vortex matter through temperature dependent NRMA signal amplitude and to (vi) reveal important information on the anomalous nature of magnetic hysteresis etc. This provides new insights to both the fundamental and technological aspects of HTS.

There are two very important observed features which need further elucidation as their origins are still not very clear though there is a consensus on the origin of NRMA - a manifestation of JJs. Firstly, how could the amplitude of the NRMA signal which is a 
manifestation of the $\mathrm{JJ}_{\mathrm{inter}} / \mathrm{JJ}_{\text {intra }}$ with markedly different temperature dependences originate in such JJs? Secondly, how could the anomalous nature of NRMA hysteresis in contrast to the normal nature of DC magnetic hysteresis under similar conditions of temperature and magnetic field is generally observed? A critical analysis of the existing understanding to explain these observed features reveals that the full explanation of these anomalies are still far from clear understanding and thus needs modifications.

Recently (Padam et al. 2006, 2010) these two aspects have been taken seriously and analyzed in a different perspective, i.e. (i) by including the role of electromagnetic interactions (EMI) for explaining the reason for undulatory temperature dependence of the NRMA signal amplitude, and (ii) by giving a special reference to energy stabilized Josephson fluxons (ESJ) for explaining the anomalous magnetic hysteresis as seen by NRMA.

The main objective of this article is to further elucidate these two problems and the importance of NRMA technique in understanding their origin in a broader and deeper perspective with inclusion of studies made on recently discovered iron pniticides. The first problem has been analyzed in more generalized way by including some more data (i.e. temperature dependence under magnetic field) so that the confusion related with the different explanations for the origin of the temperature dependence of the NRMA signal can be minimized. Similarly, the second problem based on the existing status of NRMA hysteresis and their comparison with DC magnetization hysteresis.

The samples of BSCCO based HTS used in the present study are $\left(\mathrm{Bi}, \mathrm{Pb}_{2} \mathrm{Sr}_{2} \mathrm{Ca}_{2} \mathrm{Cu}_{3} \mathrm{O}_{10+x}\right.$ which were prepared from spray dried route in addition to the earlier studied $\mathrm{Bi}_{2} \mathrm{Sr}_{2} \mathrm{Ca}_{1} \mathrm{Cu}_{2} \mathrm{O}_{8+x}$ samples with some more data and were characterized. The analysis of the results leads us to emphasize the role of EMI to explain a temperature-dependent JJs structure and to assert the role of ESJ fluxons to explain the anomalous nature of the hysteresis.

With this objective, in the section 2 of this article, the experimental details of the sample preparation and the characterization are given. In section 3, first results of work already done related with different temperature dependences of NRMA signal amplitude and the explanations given so far (3.1) are presented, then report results of present NRMA studies on sintered pellets of BSCCO samples (3.2) followed by discussion asserting the role of EMI (3.3). Similarly, section 4, will be first devoted to a brief summary of the results reported earlier on the nature of magnetic hysteresis in granular superconductors while carrying out DC magnetization and NRMA measurements (4.1) and the explanations for its origin, then report the present results (4.2) followed by discussion and emphasizing the role of energy stabilized Josephson fluxons (ESJ) in subsection 4.3. Finally, in section 5, we will summarize and give some conclusions.

Based on this exhaustive study, it is shown in this article that the inclusion of EMI and ESJ can further confirm their roles in the observed different temperature dependent NRMA signal amplitude and magnetic anomaly respectively.

In this article, the additional NRMA data showing the anomalous nature of hysteresis in some other variety of superconductors including the recently discovered iron superconductors which further confirms/supports the earlier reports makes the scenario more interesting and intriguing. 


\section{Experimental details}

The bulk pellets (diameter $=12 \mathrm{~mm}$, thickness $=2 \mathrm{~mm}$ ) of $\mathrm{Bi}_{2} \mathrm{Sr}_{2} \mathrm{CaCu}_{2} \mathrm{O}_{8+\mathrm{x}}: \mathrm{Ag}(5 \mathrm{wt} \%)$ [Bi2212] were synthesized by the matrix reaction method and the details are described earlier (Padam et al., 1999)

Whereas, the bulk pellet samples of $\mathrm{Bi}_{1.84} \mathrm{~Pb}_{0.4} \mathrm{Sr}_{2} \mathrm{Ca}_{2.2} \mathrm{Cu}_{3} \mathrm{O}_{10+y}$ : $\mathrm{Ag}$ (0 to 10 wt \%) [(Bi, $\mathrm{Pb}$ )2223] were synthesized by using spray drying method. For this, high purity powders of $\mathrm{Bi}$ $\left(\mathrm{NO}_{3}\right)_{3} .5 \mathrm{H}_{2} \mathrm{O}, \mathrm{Pb}\left(\mathrm{NO}_{3}\right)_{2}$, $\mathrm{Sr}\left(\mathrm{NO}_{3}\right)_{2}, \mathrm{Ca}\left(\mathrm{NO}_{3}\right)_{2} .4 \mathrm{H}_{2} \mathrm{O}$ and $\mathrm{Cu}\left(\mathrm{NO}_{3}\right)_{2} .3 \mathrm{H}_{2} \mathrm{O}$ were used. These powders were taken in a nominal composition of 1.84:0.4:2:2.2:3 and dissolved separately in double distilled water. Each of the individual solutions was continuously stirred with mild heating to clear all solutions and a few drops concentrated nitric acid were added, whenever needed, to fasten the process. Then all the solutions of individual powders were mixed in a beaker and labeled 'pure solution'. Different silver nitrate solutions were also made in double distilled water to obtain a silver content that corresponds to the composition of $(\mathrm{Bi}, \mathrm{Pb})-2223$ by weight percentages of $0,1,5$ and 10 . They were mixed, one by one, with the same amount of 'pure solution'. These final solutions were then kept separately in a beaker and spray dried separately to prepare the desired powders $(0-10 \mathrm{wt} \% \mathrm{Ag})$ of the above mentioned compositions. The powders so obtained were calcined separately first at $800^{\circ} \mathrm{C}$ and then at $820^{\circ} \mathrm{C}$ for one hour with one intermediate grinding. Then pellets having $12 \mathrm{~mm}$ diameter and $1 \mathrm{~mm}$ thickness, were made of each $0-10 \mathrm{wt} \%$ Ag doped (Bi,Pb)-2223 calcined powders and sintered at $835^{\circ} \mathrm{C}$ in air for 100 hours with one intermediate grinding.

Since the properties of the non- resonant microwave absorption depend to some extent on the phase purity and microstructure of the sample etc, which may vary from sample to sample even made under identical conditions, therefore, to avoid any ambiguity all the characterization studies: NRMA, X-ray diffraction (XRD), scanning electron microscope $(\mathrm{SEM}), \mathrm{EDAX}$, electrical and ac susceptibility for $\left(\mathrm{T}_{\mathrm{c}}\right)$ were done on a single sample.

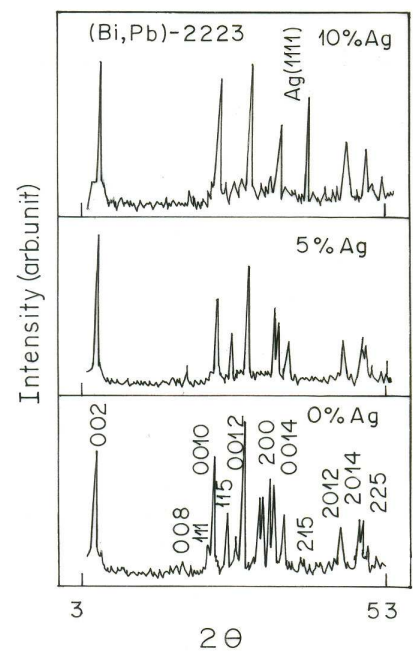

Fig. 1a. XRD patterns of (0-10wt $\% \mathrm{Ag})$ added $(\mathrm{Bi}, \mathrm{Pb})-2223$ samples showing improvement in grain alignment on increasing Ag content. 


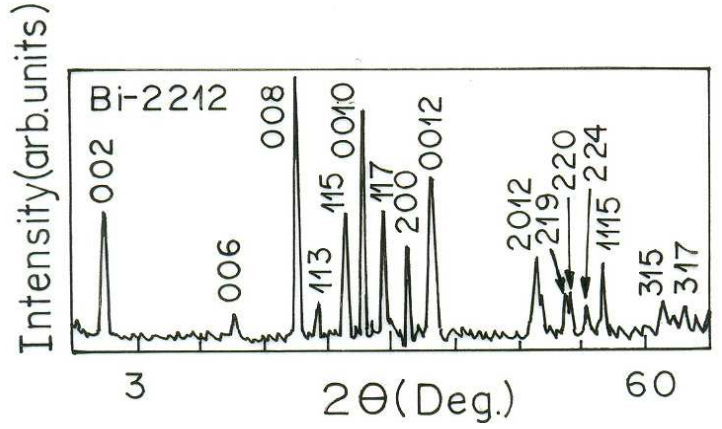

Fig. 1b. XRD pattern of powder of Bi-2212 (5wt\%Ag) sintered pellet.

Room temperature X-ray powder diffraction (Fig.1a) of the material 0, 5 and $10 \mathrm{wt} \% \mathrm{Ag}$ doped $\mathrm{Bi}_{1.84} \mathrm{~Pb}_{0.4} \mathrm{Sr}_{2} \mathrm{Ca}_{2.2} \mathrm{Cu}_{3} \mathrm{O}_{10+\mathrm{y}}$ samples, showed all these products consisted of $(\mathrm{Bi}, \mathrm{Pb})-$ 2223 phase. However, on addition of $\mathrm{Ag}$, the samples was found to improve in terms of crystallinity as revealed from the sharper peaks and directionally oriented as revealed from the increased $00 \mathrm{l}$ reflections. This indicated these contents of $\mathrm{Ag}$ addition improved the quality of the samples. Similarly, room temperature powder X-ray diffraction (Fig.1b) for the $\mathrm{Bi}_{2} \mathrm{Sr}_{2} \mathrm{CaCu}_{2} \mathrm{O}_{8+\times}(5 \mathrm{wt} \% \mathrm{Ag})$ samples established that the products consisted of (Bi-2212). Furthermore, the absence of lines associated with secondary phases like $\mathrm{Ca}_{2} \mathrm{PbO}_{4}, \mathrm{CaO}, \mathrm{CuO}$ etc. in the $\mathrm{Bi}-2212$ and in the $(\mathrm{Bi}, \mathrm{Pb})-2223$ samples indicated that the materials used in the present experiments were single phase.

A scanning electron microscopic studies of three $(\mathrm{Bi}, \mathrm{Pb})-2223(0 \mathrm{wt} \%, 5 \mathrm{wt} \%$ and $10 \mathrm{wt} \%$ Ag) samples revealed that in pure samples, there is a broad distribution of grain size and in Ag doped samples, there is a uniform grain size distribution with better oriented well connected plate like grains (not shown). Similarly, $\mathrm{Bi}_{2} \mathrm{Sr}_{2} \mathrm{CaCu}_{2} \mathrm{O}_{8+x}(5 \mathrm{wt} \% \mathrm{Ag}$ ) revealed a reasonably good microstructure with well aligned and well connected grains (not shown). Superconducting transition temperature $\left(\mathrm{T}_{\mathrm{c}}\right)$ was measured by both the electrical resistivity and ac susceptibility technique for these samples and the results are shown in (Fig.2). For the (Bi, Pb)-2223 samples $\mathrm{T}_{\mathrm{c}}$ increases from 105 to $117 \mathrm{~K}$ on increasing $\mathrm{Ag}$ content from 0 to $10 \mathrm{wt} \%$. The normal state DC resistance is lowest for the $5 \mathrm{wt} \%$ doped sample and is highest for the $10 \mathrm{wt} \%$ sample. In $5 \mathrm{wt} \% \mathrm{Ag}$ doped sample, it appears that most of the silver goes to the grain boundaries and hence is available for conduction. Therefore, minimum normal state resistance is observed in this sample. However, for the $10 \mathrm{wt} \%$ Ag doped sample, most of the silver ions go into the lattice; therefore less silver is available for normal electrical conduction, hence responsible for highest normal state resistance 


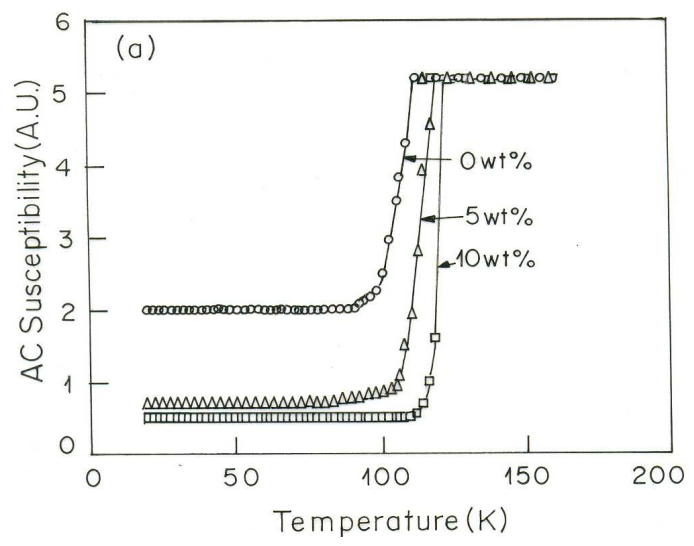

Fig. 2a. AC susceptibility dependence on temperature for silver added (0-10wt\%) samples.

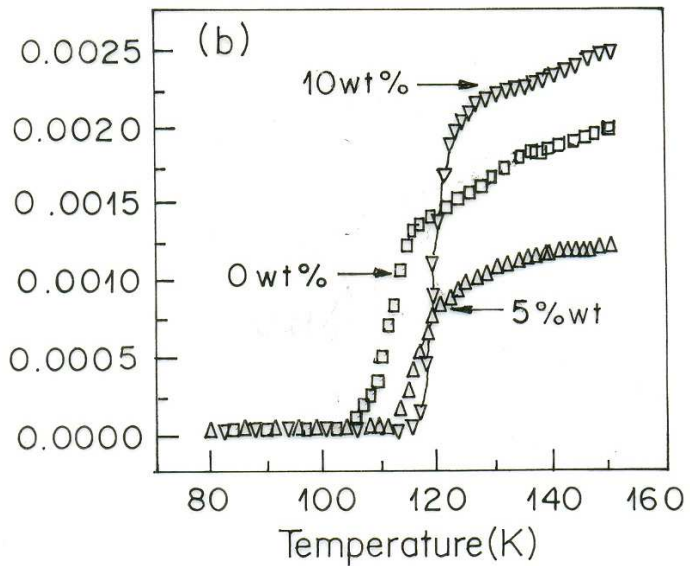

Fig. 2b. Temperature dependence of DC resistance of silver added (0-10wt\%) samples.

For the Bi-2212 sample, $\mathrm{T}_{\mathrm{c}}$ of $96 \mathrm{~K}$ was obtained (Fig .3).

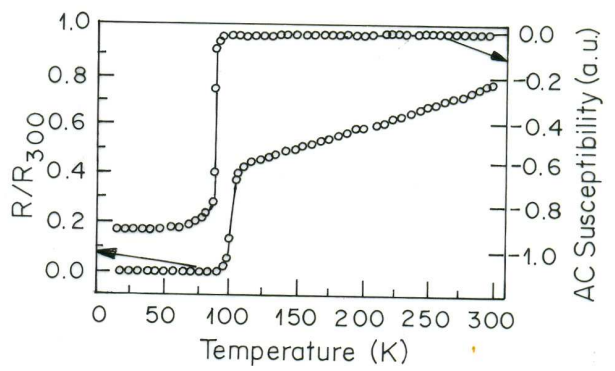

Fig. 3. Resistance and Susceptibility vs. Temperature characteristics of the Bi-2212(5wt $\% \mathrm{Ag})$ sample. 
The quality test was also confirmed by measuring ESR of the samples at room temperature and observed no ESR signal due to most expected $\mathrm{Cu}^{2+}$ ions in all the samples was observed (Fig.4). For comparison, ESR spectra of (Bi, Pb)-2223 under sintered sample (containing binary phases) which is showing $\mathrm{Cu}^{2+}$ is also depicted.

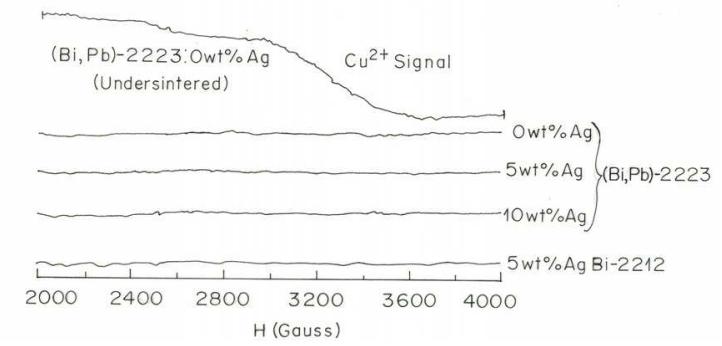

Fig. 4. EPR of (Bi, PB)-2223 and Bi-2212 samples showing absence of any signal to $\mathrm{Cu}^{2+}$ ions $\left(100 \mathrm{~mW}, \times 1.25 \times 10^{3}\right)$

All these results showing nearly single phase nature of these samples with good microstructures and sharp superconducting transitions suggested that both the samples are of a reasonably good quality. For NRMA studies, an electron paramagnetic resonance (EPR) spectrometer Bruker ER 200D X-band $(9.47 \mathrm{GHz})$ equipped with an Oxford ESR900 continuous helium gas flow temperature variation accessory was used. Details are given in reference ${ }^{7}$. The measurements were done at modulation frequency at $100 \mathrm{KHz}$ for different values of temperature $(5-300 \mathrm{~K})$ by cooling the samples in zero field and then sweeping the static magnetic field. To avoid any possibility of experimental conditions (modulation amplitude, MW power, magnetic field scan range etc.) dependent shifting, shape, phase reversal and hysteresis or other artifacts of the signal, all the measurements were done under identical conditions for each study. That is, moderate modulation amplitude of typically $4 \mathrm{G}$ and the microwave power of $20 \mathrm{~mW}$ were kept the same. Similarly, the magnetic field scan ranges were also kept the same for both the forward and reverse scans: the field was varied between the field was varied between $-50(+50)$ to $+50(-50) \mathrm{G}$. Some of the measurements were also done for high field scans from -50 to $+1050 \mathrm{G}$. It was checked and confirmed that the signal shapes are not distorted.

To see the actual variation of the NRMA signal amplitude as a function of temperature, the NRMA signal amplitude is recorded by the temperature scanning method. The NRMA signal amplitude was monitored by warming the samples from $5 \mathrm{~K}$ to $300 \mathrm{~K}$. For the Bi-2212 sample, these measurements were also done at four different fixed magnetic field values of 0,50 and 100G for each scan in order to see the effect of magnetic field on the temperature dependent NRMA signal amplitude behavior.

Signals were recorded in the derivative form [i.e. field derivative $(\mathrm{H})$ of microwave power absorbed $(\mathrm{P}): \mathrm{dP} / \mathrm{dH}$ ] resulting from magnetic field modulation of typically $4 \mathrm{G}$ peak-topeak at $100 \mathrm{KHz}$ and phase-sensitive detection technique when $\mathrm{H}_{\mathrm{mw}}$ (magnetic field component of microwaves) is perpendicularly to DC magnetic field $\mathrm{H}_{\mathrm{DC}}$. This provides information on extremely small temperature and magnetic field dependent microwave absorption $(\mathrm{P})$ changes. 


\section{Results and explanations on temperature dependent behavior of NRMA signal amplitude}

\subsection{Temperature dependent behavior of NRMA signal amplitude in different superconductors reported by other researchers}

Fig. 5 shows temperature dependence of NRMA signal amplitude reported in for different superconductors: cuprates (Blazey et al., 1987; Moorjani et al.1987; Glarum et al.1988) organic superconductors (Haddon et al., 1991) conventional superconductors ${ }^{14,17}$ ( Suss et al., 1989; Moorjani et al., 1987)etc. It is evident that markedly different variations in the NRMA signal amplitude in all these superconductors are seen on increasing temperature i.e. going from superconducting state to normal state. These different behavior mostly observed are: peak, undulatory and monotonous fall:

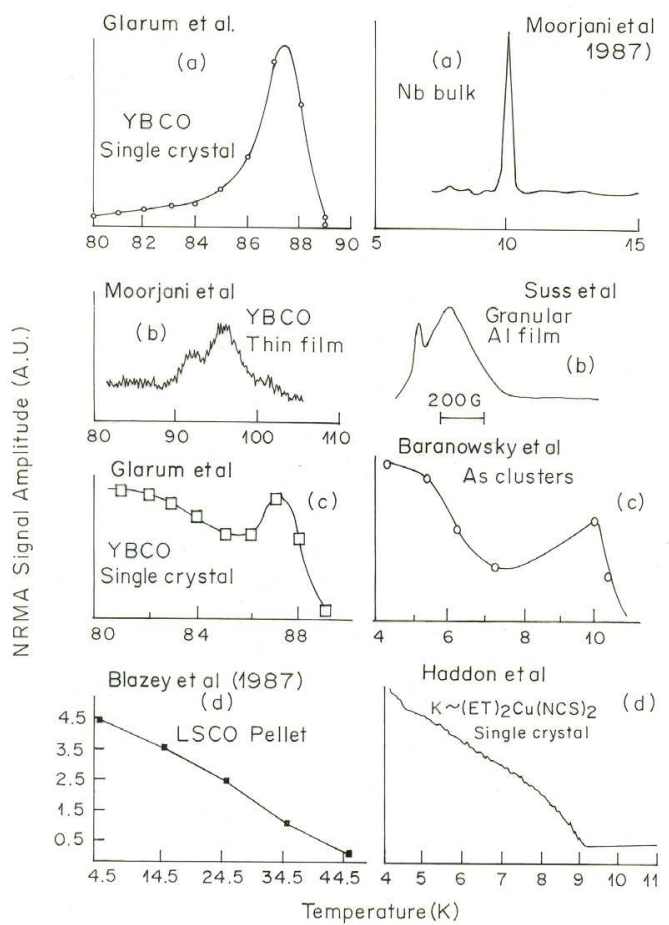

Fig. 5. Temperature dependence of NRMA signal amplitude showing different behavior irrespective of class of granular superconductor (data from literature).

i. Observation of peak/peaks in temperature vs. NRMA signal amplitude i.e. on increasing temperature, an initial exponential rise in the amplitude reaching a maximum with a subsequent exponential fall near below $\mathrm{T}_{\mathrm{c}}$ (Fig.5a), or there can be more than one peak as shown in Fig.5b.

ii. Undulatory i.e. an initial fall then a rise reaching maxima followed by a final fall (Fig.5c) on increasing temperature.

iii. A monotonous fall (Fig.5d) on increasing temperature. 
It is interesting to note that these different behavior are independent of the sample form (i.e. whether polycrystalline, thin film or single crystal) and types of superconductors. However, to realize, the full understanding of these different behavior it is necessary to clarify a considerable amount of confusion in the literature regarding the different origins of the temperature dependence of the NRMA signal amplitude by different researchers given so far which probably is due to some missing parameter. In the following, a brief account of various approaches and explanations has been given.

\subsubsection{Peak type temperature dependence}

Single peak type temperature dependence in HTS which is a characteristic feature of a sample having single superconducting phase has been well studied and its origin generally has been attributed to the different coupling strength of different JJs. So, throughout the temperature range in the superconducting state, some junctions decoupled, flluxons (JFs) penetrated and form a flux line lattice (FLL) which is controlled by Josephson interactions (JI) and dissipate power. That is, on increasing temperature, coupling strength of JJs and strength of (JI) among JFs decreases this result into an increase in number density and mobility of the fluxons penetrated within JJs. Consequently, an initial rise in the NRMA signal amplitude reaching maxima in the superconducting region at a few degrees below $T_{c}$, until most of the junctions are broken and showed weak superconductivity, hence an exponential fall in the superconducting-tonormal transition region. Above this temperature, all the JJs are broken and the material becomes normal, where the signal disappears. Details can be seen in earlier references (Portis et al., 1988, Dulcic et al., 1989)

Further, width and position of the peak have been related to a different variety of JJs. This different variety of JJs are: (i) $J_{\text {inter }}$ which dominates in sintered pellets and/or dense powders can be due to misorientation of anisotropic grains, oxygen depleted grain boundaries or secondary phases at the grain boundaries etc. and are generally thick, and (ii) $\mathrm{JJ}_{\text {intra }}$ which dominates in isolated grains can be due to twin boundaries, stacking faults or point defects etc. and are generally thin. Narrow peaks near below $T_{\mathcal{c}}$ have been assigned to a sample having a small variety of JJs which are generally thin type (i.e.Jjintra). On the other hand, broader peaks at temperatures lower than $T_{c}$ have been cited as samples having a larger variety of JJs and are more of thick type like $\mathrm{JJ}_{\mathrm{inter}}$.

It is to be noted that in contradiction to the above explanation given by majority of the researchers regarding the origin of peak type behavior assigned to temperature dependent coupling strength of different kinds of JJs, however, there are some researchers for example Moorjani et al.(1990) who still have a different opinion. That is, according to them: (i) the peak response is due to the intrinsic superconducting transition, (ii) if any NRMA signal (i.e. rising base line) appears below this peak then that is due to the presence of different JJinter. Drawback of this report is that it considers only the role of JJsinter and neglects that of JJintra even in HTS which are known to have inevitable presence of $\mathrm{JJ}_{\text {intra. }}$. Considering the role of $\mathrm{JJ}_{\text {intra }}$ e.g. due to the formation of a thin oxide layer on their $\mathrm{Nb}$ samples as reported by other researchers, can be one of explanations for the peak behavior in their $\mathrm{Nb}$ samples. Another possible reason of formation of superconducting droplets near $\mathrm{T}_{\mathrm{c}}$ giving peak response can not be ruled out. 
Like single peak which is a characteristic feature of a single superconducting phase, multiple peak behavior is a signature of multiple superconducting phases with different $\mathrm{T}_{\mathrm{c}}(\mathrm{Kataev}$ et al., 1991; Moorjani et al., 1987) in the same sample. Like the origin of single peak behavior assigned to temperature dependent coupling strength of different JJs and strength of JI in a single superconducting phase, the origin of multiple peaks is attributed to temperature dependent coupling strength of different JJs present in each superconducting phase.

\subsubsection{Undulatory and monotonous type temperature dependence}

With regard to the origin of undulatory and monotonous fall type behaviors, following different explanations have been given.

a. For the undulatory type behavior, different sources (Dulcic et al., 1990; Moorjani et al., 1990; Bohandy et al. 1988) have been reported to responsible. For example: (i) impurities, (ii) high density of defects and dominance of a variety of thick JJs etc.,(iii) to a mixture of phases with a distribution of superconducting transition temperatures and lower modulation frequency/amplitude.

b. Similarly, for a monotonous fall has been attributed to (i) impurities( Glarum et al., 1988) (ii) a deteriorated $T_{c}$ superconducting phase (Sastry et al.,1987), (iii) presence of imperfections such as stacking faults ( Bohandy et al.,1991)and (iv) a decrease in the viscosity of the fluxons on increasing temperature (Haddon et al., 1991).

It is clear that as compared to the origin of peak type response, origins of the undulatory and monotonous type behaviors are uncertain. That is, whether it is due to some impurities, imperfections, multiple superconducting / non-superconducting phases or variations in flux dynamics etc. still not clear. Further, all these reports consider the role of JI interactions only but none of them give reason for the initial fall in the signal amplitude on increasing temperature.

All these observations irrespective of the class of superconductors make this problem intriguing and motivate to investigate this discrepant data more carefully.

Recently in our earlier work (Padam et al., 2006) an attempt has been made to analyze this problem by considering the effect of electromagnetic interactions (EMI) in addition to JI and found that involving EMI can satisfactorily explain particularly the undulatory behavior.

Before going into further details, lets us see the temperature dependence of the NRMA signal amplitude in the present samples.

\subsection{NRMA signal amplitude vs. temperature behaviors in present BSCCO samples - Relevance of EMI}

\subsection{1 (Bi, Pb) samples}

Fig. 6 depicts the peak behavior for $\mathrm{Ag}\left(0,5\right.$ and10 wt \%) added $(\mathrm{Bi}, \mathrm{Pb})_{2} \mathrm{Sr}_{2} \mathrm{Ca}_{2} \mathrm{Cu}_{3} \mathrm{O}_{10+x}$ [here after called as $(\mathrm{Bi}, \mathrm{Pb})$-2223] bulk samples the temperature dependence of the signal amplitude for of constant magnetic field of $0 \mathrm{G}$ by varying the temperature (45-160K). In these experiments equal amounts of each sample and identical spectrometer operating conditions were used to facilitate comparison of the resulting spectra. It is clear that all the 
samples showing a peak behavior indicating that all the samples have a different variety of JJs. Since these results are similar to as reported by others $1-7,10,15$, therefore, its origin can also be explained on the bases of temperature dependent coupling strength of JJs / strength of JI.

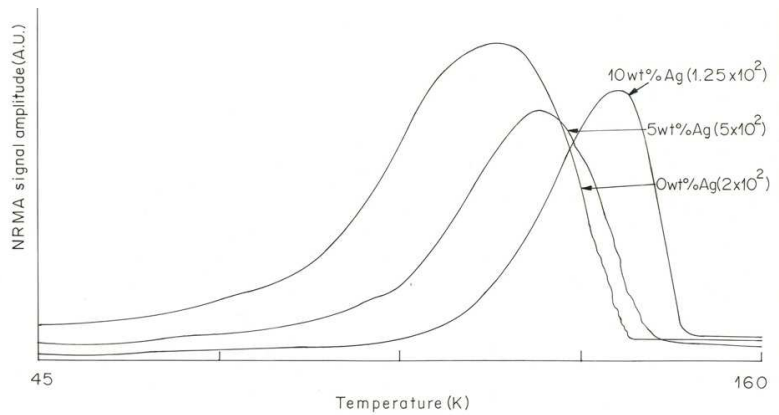

Fig. 6. Temperature of NRMA signal amplitude of Ag added (Bi, Pb)-2223 sample showing narrowing and shifting of the peaks towards higher temperature on increasing Ag content.

However, width and position of the peak was found to depend upon the amount of Ag. That is, narrowest peak at the highest temperature of $135 \mathrm{~K}$ for the $10 \mathrm{wt} \%$ Ag doped sample, whereas the broadest peak at the lowest temperature of $118 \mathrm{~K}$ for the pure sample was observed. This narrowing and shifting towards higher temperature of the peak on increasing Ag content may be due to the improvement in the quality of the sample in terms of decreasing number probably of $\mathrm{JJ}_{\mathrm{inter}}$. These results are supported by the sharper XRD peaks with enhanced (ool) reflections (Fig.2) and sharper superconducting transition and increased $\mathrm{T}_{\mathrm{c}}$ (Fig.1) on increasing Ag content. Similar results related with observation of narrow peaks at high temperatures for good quality samples and broader peaks at lower temperatures for poor quality samples have been reported by others ( Bhat et al.,1987; Portis et al.,1988; Dulcic et al.,1989; Pakulis \& G.V.Chandershekar, 1989; Sugawara et al.,2000; Kataev et al., 1991,Padam et al.,1999).

\subsubsection{Bi-2212 samples}

Fig.7a depicts an undualtory temperature dependence of the NRMA signal amplitude for the Bi-2212 (5wt \%) bulk samples kept at constant magnetic field of $0 \mathrm{G}$ and varying the temperature from $15 \mathrm{~K}$ to $105 \mathrm{~K}$ rather than the peak behavior as shown by all the three $(\mathrm{Bi}, \mathrm{Pb})-2223$ samples. This $\mathrm{Bi}-2212$ sample showed showing three distinct regions, i.e. an initial decreasing curve $\mathrm{ABC}$ (15-71.8), narrow flat $\mathrm{CD}$ (71.8-76.5K) and then a rise $\mathrm{DE}$ (76.5$82.5 \mathrm{~K})$ with maxima at around $82.5 \mathrm{~K}$ followed by subsequent exponential fall EF.

This undulatory behavior may be discussed first in the frame work of the explanation used for peak behavior. That is, on increasing temperature, strength of JI decreases (due to increasing decoupling of JJs) which results into an increase in number density and mobility of JF. This leads to an initial rise in the NRMA signal amplitude reaching a maxima at a few degrees below $\mathrm{T}_{\mathrm{c}}$, until most of the JJs are broken and showed weak superconductivity, hence an exponential fall in the superconducting - to - normal region. Above this temperature, all the JJs are broken and the material becomes normal, where the NRMA signal disappears. 
From this it is very clear that, the undulatory behavior of an initial fall in the present samples can not be explained by the temperature dependent strength of JI alone as it will lead to an initial rise with increasing temperature.

Other explanations which relates with impurities and to a mixture of phases with a distribution of superconducting transition temperatures are unlikely as the Bi-2212 samples are single phase as revealed from the observations of: (i) sharp superconducting transition from resistivity/AC susceptibility data, (ii) no reflections, corresponding to the Bi-2201 structure within the accuracy of $3 \%$ from even the powder XRD measurements and (iii) no impurity signal from EPR data (Fig.4).

Similarly another explanation which relates to the presence of high density of defects and dominance of a variety of thick JJs etc. considering only the JI is also ruled out for the obvious reason that JI alone can only explain the initial rise but not the initial fall as in the present case. This indicated that there is a need to look for some other alternative explanation and/or to include the role of some other interaction in addition to JI.

Recently the present authors (Padam et al.,2006)have given an alternative explanation for the origin of undulatory behavior by including in addition to JI, the role of electromagnetic interactions (EMI) which are not only significant but are also dominant over most of the low magnetic field and low temperature regimes, where generally the NRMA measurements are carried out. The combined effect of JI and EMI controls the FLL behavior and play an important role in absorption of microwaves. Based upon the inclusion of EMI in addition of JI, the undulatory behavior in the present Bi-2212 (5wt \%) sample has been explained as follows:

Appearance of NRMA signal at $15 \mathrm{~K}$, suggested the presence of thicker JJs (most likely JJinter ) which allow fluxons to penetrate at such low temperatures. Since density of fluxons is low at $15 \mathrm{~K}$, therefore there is a large separation among them. This leads to a weak attractive EMI. However, at this temperature, JI among fluxons is still finite. Thus combined effect of finite JI but feeble attractive EMI results into a loose packing of fluxons to form, a quasi-3D dilute liquid like FLL (i.e. disordered phase) in which the fluxons are mobile. An increase in temperature causes breaking of more number of $J_{\mathrm{inter}}$ and allows more number of fluxons to penetrate, thus reduces the inter-fluxon distance. This in turn decreases strength of JI but increases that of the EM attractions among the fluxons. Thus are able to suppress their even increasing thermally activated fluxon motion as a result of raising temperature. This trend continues up to $71.8 \mathrm{~K}$ and results in the decrease of MWA, hence, a decrease in the signal amplitude.

On further increasing temperature, due to continuation of the above trend, probably, the EM attraction becomes which minimize the thermally activated fluxon motion and results in weak temperature dependence of the amplitude in the narrow temperature range (71.8$76.5 \mathrm{~K})$. In this range, a phase transition from quasi-3D dilute liquid like FLL to a quasi-3D solid like FLL (ordered phase) probably also occur.

However, above $76.5 \mathrm{~K}$ fluxon density increases to an extent that the inter-fluxon EM repulsion overpowers inter-fluxon EM attraction and the system now becomes more sensitive to increasing thermal fluctuations. Due to dominance of both the EM repulsion and increasing thermal fluctuations, fluxon motion is more prevented and the FLL starts melting and transits into a quasi-2D dense liquid. This leads to an increase in MWA, hence a rise in the amplitude with a peak at around $82.5 \mathrm{~K}$, where it melts and leads to a fall in MWA in $82.5-105 \mathrm{~K}$. 
From the above one can say that with the inclusion of EMI the undulatory particularly the initial fall portion has been explained which otherwise was unexplainable with taking JI alone. Based upon this explanation of inclusion of EMI in addition to JI, the undulatory temperature dependence of NRMA signal amplitude as reported by others in superconducting sample having thick JJinter can also be explained. To confirm, the measurements were done by keeping the sample at a constant magnetic field as is known that the magnetic field suppresses JJinter response. The results obtained for two fields of 50G and 100G are shown in Figs.7b, 7c. Interestingly, for both the fields, the undulatory behavior which was obtained at $0 \mathrm{G}$ disappeared and instead a peak response obtained and the peak gets narrowed on increasing magnetic field. This clearly suggested that the initial fall is related with the presence of thicker JJinter which play a dominating role at low temperatures.

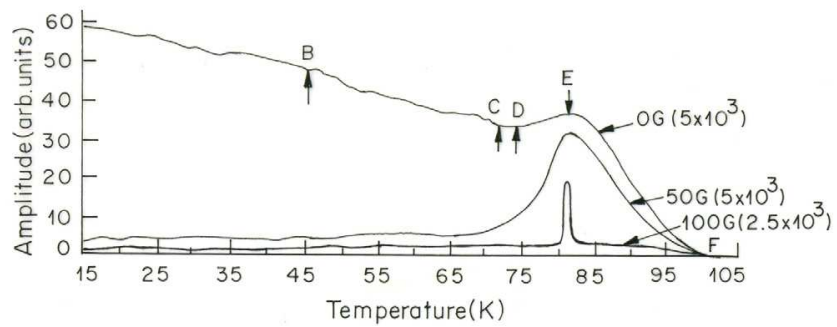

Fig. 7. NRMA signal amplitude vs. temperature curve for Bi-2212 (5wt\%Ag) sample in OG, $10 \mathrm{G}$ and 50G.

Based upon this explanation, all the results reported in literature showing undulatory can also be explained on the basis of presence of thick JJs which dominate at low temperature and lead to rising strength of EMI initially, hence an initial fall in the NRMA signal amplitude on increasing temperature.

Similarly, the monotonous fall in the NRMA signal amplitude might also be due to the dominance of thicker $\mathrm{JJ}_{\text {inter }}$ (which can be due to impurities, impurity phases, defects etc.) which allows fluxons to enter at low temperatures and increase the probability of EMI among fluxons, thereby decreasing the mobility of the fluxons hence the NRMA signal amplitude with increasing temperature.

From the above one can say that if a superconductor has more of multitude of thick JJinter, then the strength EMI overpowers that of JI and generally show undulatory or monotonous response on increasing temperature.

In the next section the second equally important results related with anomalous nature of NRMA hysteresis are given.

\section{Results and explanations on anomalous nature of magnetic hysteresis behavior of NRMA}

It would be appropriate to give a brief account of earlier reports related particularly with the nature of NRMA hysteresis in comparison to that of the DC magnetization method and its universality in different granular superconducting systems including the present results before any elaborative discussion related to its origin. 


\subsection{A comparative data on the nature of DC and NRMA magnetic hysteresis reported by other researchers}

Fig. 8 shows a comparison of set of data available in literature in single crystals of YBCO (Dulcic et al. 1990; Pakulis \& G.V.Chandershekar, 1989) and Bi-2212 (Pradhan et al., 1995) of these two measurements under almost similar experimental conditions of temperature, magnetic field scan and orientation of the magnetic field with respect to crystal axis. It is worth noting that for both the $\mathrm{YBCO}$ and $\mathrm{Bi}-2212$ systems, the decreasing $\mathrm{H}$ curve lying above the increasing $\mathrm{H}$ curve observed in DC magnetization, the decreasing $\mathrm{H}$ curve lies below the increasing $\mathrm{H}$ curve nature of hysteresis shown by NRMA method. This indicated that in contrast to the normal nature of hysteresis observed by DC method, the nature of hysteresis shown by NRMA is anomalous under similar experimental conditions of temperature, magnetic field.

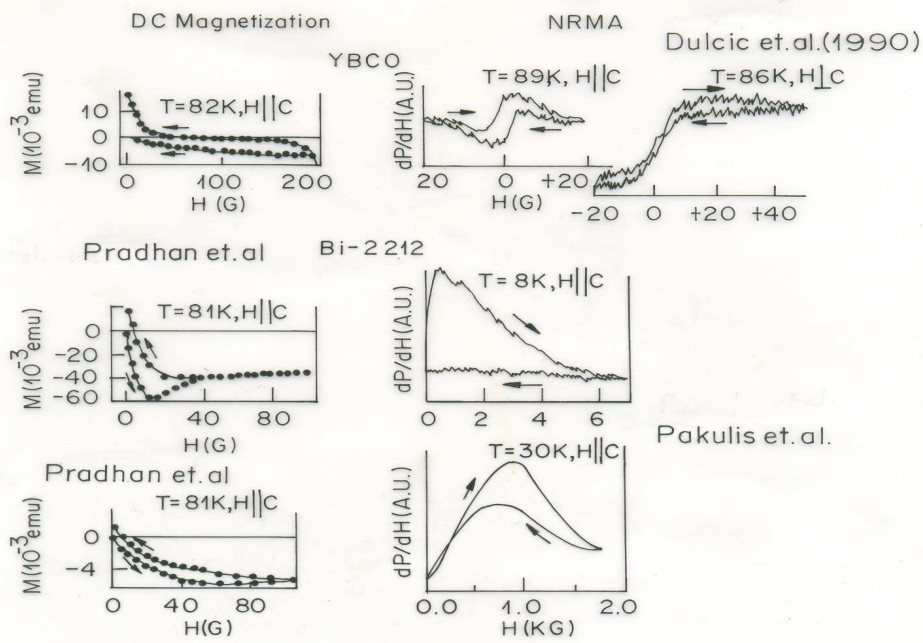

Fig. 8. Comparison of NRMA and DC magnetic hysteresis in YBCO/Bi-2212 single crystals showing the anomalous hysteresis as seen in NRMA in contrast to normal in the DC magnetization (data from literature).

\subsection{Magnetic hysteresis of NRMA in different superconductors from unconventional to conventional observed by other researchers}

Fig.9 exhibits the results of NRMA hysteresis reported earlier in different superconducting systems like: unconventional intrinsically granular cuprates (Blazey et al.,1988),borocarbides (Kadam et al.,1994) fullerene ( Zakhidov et al.1991) $\mathrm{MgB}_{2}$ ( Joshi et al.,2002) Iron pniticides (Panarina et al. 2010)and granular conventional type I/type II superconductors ( Baranowsky et al., 1991; Mahel \& S.Benacka,1992)) It further confirms that irrespective of the system of the superconductor, in all the NRMA curves, the decreasing $\mathrm{H}$ lies below the increasing $\mathrm{H}$ curve at temperatures much below $\mathrm{T}_{\mathrm{c}}$ and in low magnetic field regions. This clearly indicated anomalous hysteresis nature at LT/LH in different superconducting systems from unconventional to conventional while carrying out NRMA measurements. 


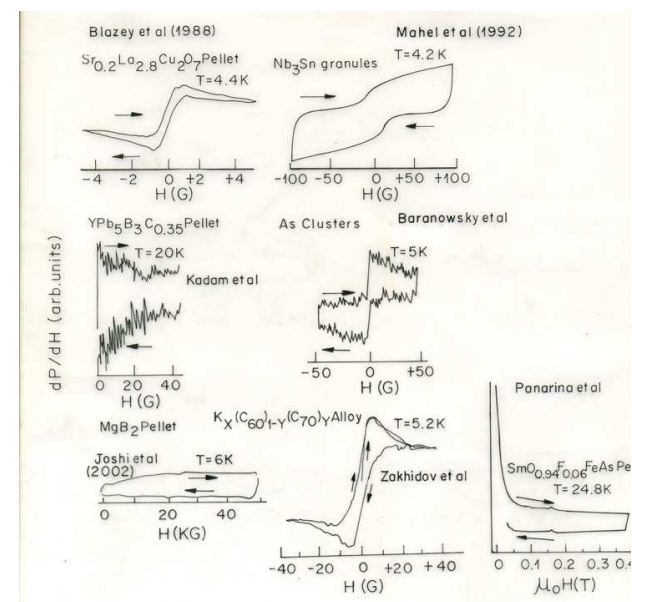

Fig. 9. NRMA hysteresis in different superconductors (data from literature) showing anomalous hysteresis irrespective of class of granular superconductor.

\subsection{NRMA hysteresis in the present BSCCO samples}

$\mathrm{dP} / \mathrm{dH}$ vs. $\mathrm{H}$ curves of NRMA signals recorded for the present Bi-2223 samples in the high field scans for both forward $(-50$ to $+1025 \mathrm{G})$ directions at temperatures in the range of 5.5 to $135 \mathrm{~K}$ were taken and the results for $90 \mathrm{~K}$ are shown in Fig.10a. Two signals: one the usual near zero field signal and another a hump like feature (encircled) which varies in amplitude and width with increasing temperature along with the former signal have been observed. Similar observations of zero field signal along with this kind of feature (encircled) have been reported earlier by some researchers including present authors (Padam et al. 2010) and are also shown in Fig. 10b for Bi-2212 single crystal (rf) (Baginskii et al. 1994, rf absorption studies) and in Fig.10c for Tl-2212 single crystal (NRMA studies) (Joshi et al. 2000, MW absorption studies)The former well known zero field signal is due to viscous motion of intergranular (IGBJ)/intragranular (IG) fluxons. Whereas the latter hump like feature is due to fluxons generated due to tilt motion of flux lattice (Brandt, 1991; Kleiner et al. 1992) It is these fluxons which have been considered to be responsible for the general anomalous magnetic hysteresis and are named as ESJ in our earlier publication (Padam et al. 2010)

Similar results were obtained in Bi-2212 samples. Fig.11 depicts low field forward (-50 to $+50 \mathrm{G})$ and reverse field (+50 to $-50 \mathrm{G}$ ) magnetic sweeps of the NRMA signals in the both the Bi-2223 and Bi-2212 samples showing hysteresis at LT $(5.5 \mathrm{~K}, 20 \mathrm{~K})$ and at HT $(110 \mathrm{~K}$, $80 \mathrm{~K})$. It can be seen that the decreasing field curve lies below the increasing field curve for both the samples not only at HT but also at LT. This indicated that the nature of NRMA magnetic hysteresis is anomalous in the present samples even at low temperatures as low $5.5 \mathrm{~K}$.

Thus all the above data reported earlier and in the present work confirms the anomalous nature of hysteresis in different granular superconductors including recently discovered iron pnictides under LT/LH conditions as seen by the NRMA method. 

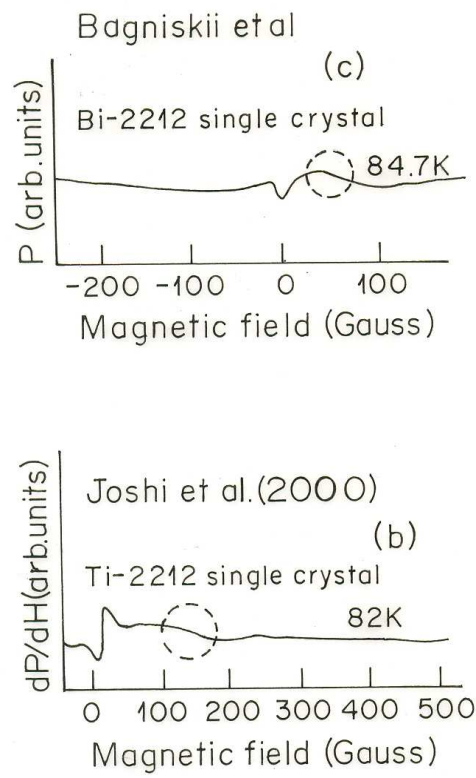

(a)

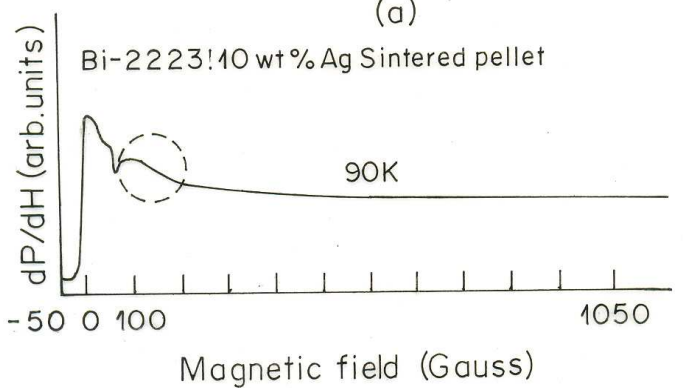

Fig. 10. $\mathrm{dP} / \mathrm{dH}$ as a function of magnetic field showing signal (encircled) due to ESJ fluxon 

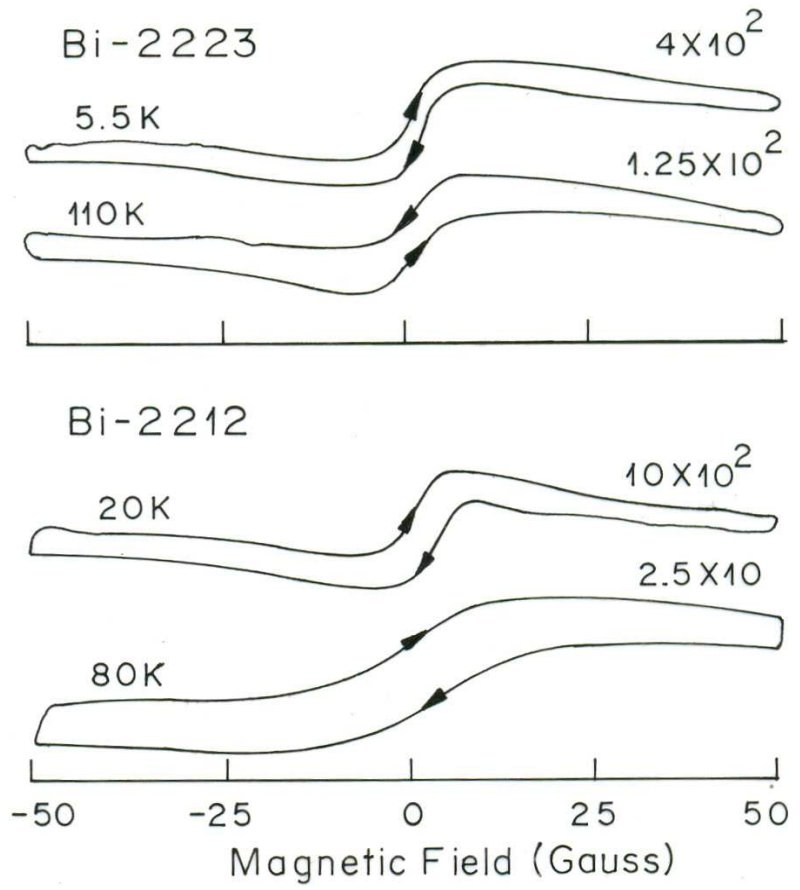

Fig. 11. NRMA hysteresis in present BSCCO sintered pellets of LT and at HT.

\subsection{Origin of NRMA anomalous magnetic hysteresis and the relevance of ESJ fluxons}

Before elaborating more on the origin of anomalous hysteresis, let us give a brief account of the development of the understanding related with the observations of different nature of hysteresis depending upon the (i) class of superconductors (i.e. conventional or unconventional), (ii) experimental conditions of temperature $(\mathrm{T})$ and magnetic field $(\mathrm{H})$ and (iii) the sensitivity of the method (DC magnetization methods: DC magnetic field $\left(\mathrm{H}_{\mathrm{DC}}\right)$ variation of (i) flux density (B), (ii) microwave surface resistance (Rs) and (iii) transport critical current density [J $\mathrm{J}_{\mathrm{c}}$ ( $\mathrm{Ji}$ et al. 1993)and of modulated magnetic field variation of non-resonant microwave power absorption (P) NRMA technique( Czyzak \& J.Stankowski, 1991).

That is, (i) normal nature of hysteresis in conventional superconductors at all temperatures and/or magnetic field by any measurement method, (ii) normal hysteresis at low temperatures (LT) and/or low magnetic field (LH) and anomalous hysteresis at high magnetic field $(\mathrm{HH})$ and/or high temperatures (HT) by in granular conventional / unconventional superconductors by DC magnetization and microwave methods and (iii) anomalous hysteresis not only at HH /HT but also at LH/LT in granular conventional / unconventional superconductors while carrying out NRMA measurements.

The normal nature of hysteresis under any conditions conventional superconductors has been very well explained by the Beans model (Beans, 1962) which considers the role of 
intragranular Abrikisov/Pancake fluxons (IG). The anomalous nature of hysteresis at $\mathrm{HT} / \mathrm{HH}$ in granular conventional/ unconventional superconductors has been well explained by Ji et al model by modifying Beans model on considering the role of intergranular Josephson (IGBJ) fluxons in addition to IG.

It is surprising that despite the voluminous literature on the NRMA hysteresis; only a few groups (Sugawara et al. 2000; Padam et al. 1991; Czyzak \& J.Stankowski, 1991; Mahel et al., 1992; Ramachandran et al. 1994) have mentioned about its anomalous nature and related it to Ji et al model and its variant by Mahel et al. and Ramachandran et al. but they have their own drawbacks as will be clear below.

The present/ others results on the presence of anomalous magnetic hysteresis can be discussed in the frame work of the most successful model by Ji et al.which explain HT/HH anomalous hysteresis in granular superconductors. According to this model, there are two kinds of fluxons: intergranular (IGBJ) and intragranular (IJ) in granular superconductors. Further, at $\mathrm{HT} / \mathrm{HH}$, due to the higher number density and higher mobility of the IGBJ fluxons to those of the IJ fluxons, the magnetization during increasing field curve is lower than that during decreasing field curve and gives anomalous hysteresis. Although this model successfully explains the anomalous hysteresis at $\mathrm{HT} / \mathrm{HH}$, however the observed anomalous hysteresis at LT/LH in the present samples and reported by others can not be explained.

Further, these results also cannot be explained even by using variant of Ji et al. model by Mahel et al. and Ramachandran et al. particularly used for explaining NRMA anomalous hysteresis. This is because, the observation of anomalous magnetic hysteresis in the present samples as well as reported by others is not limited by the conditions of imposed by these models. For example, the former model includes the effect of modulation amplitude threshold field on the distribution of both the intergranular Josephson (IGBJ) and intragranular (IJ) fluxons). However, this model is applicable only for a limited modulation amplitude range. Similarly, the latter model which includes the effect of modulation amplitude threshold field on the distribution of flux suffers from its stringent requirement of threshold field value.

In our recent work (Padam et al., 2010) an attempt has been made to solve this problem by using another variant of Ji et al. model by including the role of Energy Stabilized Josephson (ESJ) fluxons in addition to IGB/IG fluxons and highlighting the features of the extremely sensitive NRMA method which makes it unique in creating and detecting a situation for the appearance of anomalous hysteresis. It was found that by combining the role of ESJ fluxons with the uniqueness of the NRMA technique, it is possible to explain the observation of LT/LH anomalous hysteresis too.

It is known that apart from IGBJ fluxons and IJ fluxons, there exists another kind of Josephson fluxons which are generated as a result of thermal/Lorentz forces induced tilt motion of flux lattice (Brandt, 1991; Kleiner et al. 1992) It is these fluxons are ESJ fluxons which not only increase the total number of more mobile fluxons at LT and/ LH, etc but also are detected due to the extra sensitivity of the NRMA method (Khachaturyan et al., 1987), thereby revealing the anomalous hysteresis. 
Before proceeding further, it is essential to essential to highlight the experimental features of NRMA method, which makes it unique. For example, its ability (i) in creating a situation for enhanced ESJ fluxons,(ii) in detecting even minute changes in the fluxon density and (iii) in distinguishing between different types of fluxons, where all other DC magnetization techniques unable to do so.

In this context it is known that NRMA technique uses ESR spectrometer, where DC magnetic field $\left(\mathrm{H}_{\mathrm{DC}}\right)$ is modulated with a modulation field $\left(\mathrm{H}_{\mathrm{m}}\right)$ superimposed parallel on $\mathrm{H}_{\text {DC }}$. Due to this $\mathrm{H}_{\mathrm{m}}$ of magnetically modulated hysteresis are observed unlike direct field hysteresis measurements. This modulation field in addition to $\mathrm{H}_{\mathrm{DC}}$ (used in B vs.H and JC vs. $\mathrm{H}$ methods) and $\mathrm{H}_{\mathrm{DC}}+\mathrm{H}_{\mathrm{mw}}$ (both used in Rs vs. $\mathrm{H}$ method) gives a few advantages. For example, it provides extra energy (due to a combined effect of three fields: $\mathrm{H}_{\mathrm{DC}}+\mathrm{H}_{\mathrm{mw}}+\mathrm{H}_{\mathrm{m}}$ ) required to overcome the strength of the Josephson junctions; as a result, IGBJ/IGJ fluxons enter at much lower temperatures/low magnetic fields. Another is a stronger Lorentz force, particularly due to $\mathrm{H}_{\mathrm{m}}$ induced oscillatory currents, which tilt the IGBJ/IGJ fluxons, and probably becomes a volume source of generation of ESJ fluxons. Further, a combination of the modulation field in conjunction with phase sensitive makes ESR an extremely sensitive and powerful tool to detect any feeble change and to distinguish between different types of fluxons (Portis et al.1988; Dulcic et al.1897, 1988, 1989). Furthermore, the sensitivity of the NRMA method, which uses ESR, is even five orders of magnitude greater than the limit of the ESR spectrometer (Khachaturyan et al. 1987).

All these parameters make ESR technique an extra sensitive and unique in comparison of other techniques. Therefore, any change however minute and at times seemingly intractable, related with density/distribution/motion and different types of fluxons depending upon temperature/magnetic field/sample form etc. can be easily detected in NRMA. Furthermore, NRMA is the only method (Portis et al.1988; Dulcic et al.1897, 1988, 1989) which is able to distinguish between above mentioned different types of fluxons, i.e.IGBJ, IGJ, IG and ESJ etc.

Using this explanation, the observed anomalous nature of hysteresis in the present samples (which also shows the signal due to ESJ fluxons) and reported in a variety of superconductors can be explained.

\section{Summary and conclusions}

The present NRMA studies made on Bi-2223 / Bi-2212 samples and similar studies by other researchers in a variety of superconductors from conventional to unconventional have been reported. Main focus has been given on two most important aspects. First is the different temperature dependences of the NRMA signal amplitude observed by us and by others. The second one is on the observation of anomalous magnetic hysteresis in the present samples and in reports of others. These studies showing different types of behaviors: peak type undulatory and monotonous temperature dependent signal amplitude behavior and the anomalous magnetic hysteresis at all temperatures below $T_{c}$ have been analyzed and discussed by taking into consideration of parameters/interactions not envisaged before. 
From the above studies and discussion, it comes out that the undulatory type/ monotonous temperature dependent behavior of NRMA signal amplitude in a variety of granular superconductors can be very well explained if the role of EMI is included with JI.

In addition, the presence of additional fluxons (ESJ) in the present Bi-2223 / Bi-2212 samples and in other granular superconductors at all temperatures which probably formed due to a combined effect of additional fields like oscillatory MW field and modulation field are responsible for the anomalous hysteresis while carrying out NRMA measurements.

\section{Acknowledgements}

The author is grateful to Prof.R.C.Budani, Director, and National Physical Laboratory for his constant encouragement during the course of this work. The author is highly indebted to Prof.S.V.Bhat (IISC, Banglore) and Prof.M.R.Tripathy (Dept.Of Electrical and Communications Engng. ASET, Noida) for NRMA experiments. The author is highly grateful to Dr.S.N.Ekbote for his guidance and valuable suggestions.

\section{References}

Baginskii, K.V., Berezin, V.A., Govorkov, S.A. and Tulin, V.A. (1994).Sov.Phys.JETP, 160, 60.

Baranowsky, J.M.,Lilientel, W.Z., Yan W.F. and Weber, E.R. (1991).Phys. Rev. Lett. 66, 3079 .

Beans, C.P. (1962) Phys.Rev.Lett, 8, 253.

Bele, P., Brunner, H., Schweitzer, D. and Keller, H.J. (1994) Solid State Communications 92,189.

Bhat, S.V., Ganguly, P and Rao, C.N.R. (1987) Pramana-J. Physics 28, 2425.

Blazey, K.W., Muller, K.A., Bednorz, J.G., Berlinger, W., Amoretti, G., Buluggiu, E., Vera A.and Matacotta, F.C. (1987) Phys. Rev. B36, 7241.

Blazey, K.W., Portis, A.M.and Bednorz, J.G. (1988) Solid State Commn.56, 1153.

Blazey, K.W., Portis, A.M.and Holtzberg, F.H. (1989) Physica C157, 16.

Bohandy,J.,Kim, B.F., Adrianand F.J., Moorjani, K. (1989)Phys.Rev.B 39,2733.

Bohandy, J., Kim, B.F., Adrianand F.J., Moorjani, K., Arcangelis S.D. and Cowan, D.O., (1991) Phys.Rev.B43, 3724.

Brandt, E.H. (1991) Phys.Rev.Lett.67, 2219.

Czyzak B. and Stankowski, J. (1991) Phys. Stat. Sol. (b) 166, 219.

Dulcic, A., Leontic, B., Peric, M.and Rakvin, B. (1989) Europhys.Lett.,10,593 Dulcic,A.,Crepeau,R.H.,Freed,J.H.,Schneemeyean,L.H.and Waszezak,J.V.(1990) Phys.RevB.42,2155.

Gittleman J.I.and Rosenblum, B. (1966) Phys. Rev.Lett. 16, 734.

Glarum, S.H., Marshall, J.H.and Schneemeyer, L.F. (1988) Phys.Rev.B 37, 7491.

Haddon, R.C., Glarum, S.H., Chichester, S.V., Ramirez, A.P. and Zimmerman, N.M. (1991) Phys.Rev.B. 43, 2642.

Hashizume, A., Yamada, J., Kohmoto, H., Yamada, Y. Endo T. and Shahabuddin, M. (2001) Physica C 357-360,481.

Indovina, P.L., Onoti, P. and Tabet, E. Solid State Commun. (1970) 8, 1721. 
Ji, L., Rzchowski, M.S., Anand, N. and Tinkham, M. (1993) Phys.Rev.B47,470.

Joshi, J.P., Anand, A., Bhat, S.V., Chowdhary P.and Bhatia, S.N. (2000) Physica C 341-348, 1229.

Joshi, J.P., Sarangi, S., Sood, A.K., Pal, D.and Bhat, S.V. (2002) Parman, J.Phys. 58,361.

Kadam, R.M., Sastry, M.D., Hassain, Z., Mazumdar, C., Nagarajan, R., Gupta, L.C.,Godart,C.andVijayaraghvan,R.(1994)PhysicaC,232,359.

Kadam, R.M., Wani, B.N., Sastry M.D.and Rao, URK, (1995) Physica C, 246,262.

Kataev, V., Knauf, N., Buchner B.and Wohlleben, D. (1991).Physica C 184, 165.

Khachaturyan, K., Weber, E.R., Tejedor, P., Stacy Aand A.M.Portis, (1987) Phys.Rev. 36, 8309 Kheifets, A.S. and Veinger, A.I. (1990) Physica C 165, 491.

Kim, Y.W., deGraaf, A.M., Chen, J.T., Friedmanand E.J., Kim, S.H. (1972).Phys, .Rev.B 6,887

Kleiner, R., Steinmeyer, F., Kunkel, G. and Muller, P.Phys.Rev.Lett. (1992) 68, 2394.

Mahel, M.and Benacka, S. (1992a) Solid State Commun. 83,615.

Mahel, M., Adam, R., Darula. M., Chromik S. and Benacka, S. (1992b) Physica C, 202,243.

Moorjani, K., Bohandy, J., Adrian, F.J., Kim, B.F., Shull, R.D., Chiang, C.K., Swartzendruber L.J.and Bennet, L.H. (1987) Phys. Rev.36, 4036.

Moorjani, K., Bohandy, J., Kim B.F.and Adrian, F.J. (1990). Solid State Commun. 74,497.

Muller, K.A., Pomerantz ,M.and Knoedler, C.M. (1980) Phys.Rev.Lett.45,832.

Padam, G.K., Ekbote, S.N., Tripathi, R.B., Sharma, M., Suri, D.K., Rao, S.U.M., Rao, Nagpal, K.C.and Das, B.K. (1991).Solid State Commun,40,271.

Padam, G.K., Ekbote, S.N., Tripathi, M.R., Srivastava, G.P. and Das, B.K. (1999) Physica C 315,45 .

Padam,G.K., Ekbote, S.N., Sharma, M., Tripathi, M.R., Srivastava, G.P.and Das, B.K. (2006) Jap.J.Appl.Phys., 45,No.1A,84.

Padam, G.K., Arora N.K.and Ekbote, S.N. (2010) Materials Chem.Phys.123, 752.

Pakulis E.J.and Chandershekar G.V., (1989) Phys. Rev.B39, 808.

Panarina,N.Y.,Talanov, Y.I., Shaposhnikova, T.S., Beysengulov, N.R., Vavilona,G.,Behr, E., Kondrat, A., Hess, C., Leps, N., Wurmehl, S., Klinger, R., Kataev, V.and Büchner, B. (2010) Phys. Rev. B 81, 224509.

Pascher, N., Deisenhofer, J., Krug von Nidda, H.-A., Hemmida, M. , Jeevan, H., Gegenwart, S. P. and Loid, A. (2010) Phy. Rev.B 82, 054525.

Peric, M.,Rakvin,B. Prester, M., Brnicevic, N.,Dulcic, A(1988) Phys.Rev. B37, 522.

Portis, A.M., Blazey, K.W., Muller K.A.and J.G.Bednorz (1988)Europhys. Lett. 5, 467.

Pradhan, A.K., Roy, S.B., Chaddah, P., Chen, C. and Wanklyn, B.M. Phys.Rev.B (1995) 52, 6215.

Ramachandran, J.S., Huang, M.X., Bhagat, S.M.(1994) Physica C,234,173.

Roberts, J., Petit, P., Yildirim, T and Fischer, J.E. (1998-II) Phys. Rev B 157, 1226.

Saharangi , S.and Bhat, S.V.http://arxiv.org/fH/cond-Mat/papers/0511701.pdf

Sastry,M.D., I.Dalvi, A.G., Babu, Y., Kadam, R.M., Yakhmi, J.V. and Iyer, R.M. (1987) Nature, 330, 49 .

Sugawara, K., Arai, N. Kouzuki, A., Ichimura, S., Naoi, H., Hotta K.and H.Hirose, (2000) Inter.J.Modern PhysicsB14, No.16, 1633.

Suss, J.T., Berlinger, W., Portis, A.M., Muller, K.A., Jeanneret B.and Martinoli, P. (1989) Solid State communications, 71,929. 
Veinger, A.I., Zabrodskii A.G. and Tisnek, T.V. (1995).Supercond.Sci.Technol. 8,368.

Zakhidov, A.A., Imaeda, K., Ugawa, A., Yakushi, K., Inokuchi, H., Iqbal, Z., Baughman, R.H., Ramakrishana B.L.and Achiba, Y. (1991) Physica C 185,411. 


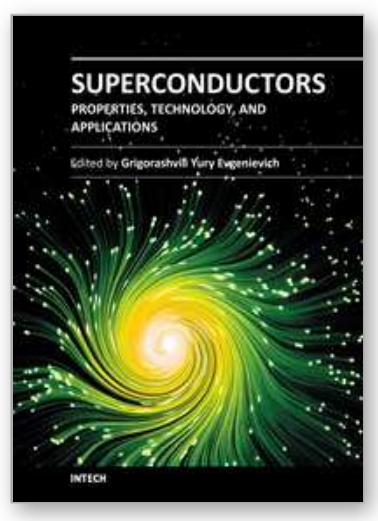

\author{
Superconductors - Properties, Technology, and Applications \\ Edited by Dr. Yury Grigorashvili
}

ISBN 978-953-51-0545-9

Hard cover, 436 pages

Publisher InTech

Published online 20, April, 2012

Published in print edition April, 2012

Book "Superconductors - Properties, Technology, and Applications" gives an overview of major problems encountered in this field of study. Most of the material presented in this book is the result of authors' own research that has been carried out over a long period of time. A number of chapters thoroughly describe the fundamental electrical and structural properties of the superconductors as well as the methods researching those properties. The sourcebook comprehensively covers the advanced techniques and concepts of superconductivity. It's intended for a wide range of readers.

\title{
How to reference
}

In order to correctly reference this scholarly work, feel free to copy and paste the following:

G.K. Padam (2012). Non Resonant Microwave Absorption (NRMA) Anomalies in High Temperature Superconductors (HTS) Relevance of Electromagnetic Interactions (EMI) and Energy Stabilized Josephson (ESJ) Fluxons, Superconductors - Properties, Technology, and Applications, Dr. Yury Grigorashvili (Ed.), ISBN: 978-953-51-0545-9, InTech, Available from: http://www.intechopen.com/books/superconductors-propertiestechnology-and-applications/non-resonant-microwave-absorption-nrma-in-superconductor-relevance-ofelectromagnetic-interactions

\section{INTECH}

open science | open minds

\section{InTech Europe}

University Campus STeP Ri Slavka Krautzeka 83/A

51000 Rijeka, Croatia Phone: +385 (51) 770447

Fax: +385 (51) 686166

www.intechopen.com

\author{
InTech China \\ Unit 405, Office Block, Hotel Equatorial Shanghai \\ No.65, Yan An Road (West), Shanghai, 200040, China \\ 中国上海市延安西路65号上海国际贵都大饭店办公楼405单元 \\ Phone: +86-21-62489820 \\ Fax: $+86-21-62489821$
}


(C) 2012 The Author(s). Licensee IntechOpen. This is an open access article distributed under the terms of the Creative Commons Attribution 3.0 License, which permits unrestricted use, distribution, and reproduction in any medium, provided the original work is properly cited. 